\title{
On the spin-up events and spin direction of the X-ray pulsar GX 301-2
}

\author{
Jiren $\operatorname{Liu}^{1 \star}$ \\ ${ }^{1}$ National Astronomical Observatories, 20A Datun Road, Beijing 100012, China
}

\begin{abstract}
Recently a retrograde neutron star is proposed for the classical wind-fed X-ray pulsar, GX 301-2, to explain the orbital spin-up to spin-down reversal near periastron, based on the stream model invoked to explain the pre-periastron flare of GX 301-2 previously. We study in detail three rare spin-up events detected by Fermi/GBM and find that the spin derivatives are correlated with the Swift/BAT fluxes, following a relation of $\dot{\nu} \propto F^{0.75 \pm 0.05}$. All the spin-up events of GX 301-2 started about 10 days after the periastron, which is the time needed for tidally stripped gas to reach the neutron star. The slow rotation of the optical companion implies that the accreted matter is likely to have angular momentum in the direction of the orbital motion, as in a Roche-Lobelike overflow. As a result, the spin-up events of GX 301-2 would favor accretion of a prograde disk to a prograde neutron star. We also find that the flare of intrinsic X-ray emission of GX 301-2 happened 0.4 days before periastron, while the flare of low energy emission (2-10 keV) happened about 1.4 days before periastron. The preceding low energy flare can be explained by stronger absorption of the intrinsic X-ray emission closer to the periastron. This finding weakened the need of the stream model. The pulse fraction of GX 301-2 near periastron is reduced heavily, which is likely caused by Compton scattering process. Compton reflection from the optical companion might be responsible for the observed orbital spin reversal of GX 301-2.
\end{abstract}

Key words: Accretion -pulsars: individual: GX 301-2 - X-rays: binaries - stars: rotation

\section{INTRODUCTION}

In accretion-powered X-ray pulsars, the spinning neutron star with a strong magnetic field accretes material from the optical companion star and produces pulsating X-ray emission. The mass transfer process could be ' through either a stellar wind or a Roche-Lobe overflow ' or a combination of both. The transport of angular momentum to the neutron star leads to variations of the spin frequency measurable on days timescale, and therefore, the spin history of X-ray pulsar can be used to probe the mass transfer process (e.g. Rappaport \& Joss 1977; Ghosh \& Lamb 1979; Wang 1981). Some X-ray pulsars show spin-up/spin-down reversals separated by intervals from days to decades, a puzzling phenomena not well understood (e.g. Makishima et al. 1988; Chakrabarty et al. 1997; Bildsten et al. 1997; Malacaria et al. 2020).

Recently, Mönkkönen et al. (2020) proposed a retrograde neutron star for the classical wind-fed X-ray pulsar, GX 301-2, which has a long pulse period $\sim 680 \mathrm{~s}$

^ E-mail: jirenliu@nao.cas.cn
(White et al. 1971). While retrograde neutron stars have been suggested to be reminiscent of the natal kick during the supernova explosion of the progenitor Hills 1983; Brandt \& Podsiadlowski 1995), the spin direction of neutron star is generally hard to constrain observationally. The GX 301-2 system has a binary period $\sim 41.5$ days and an orbital eccentricity $\sim 0.46$ (Sato et al. 1986; Koh et al. 1997). Its optical companion, Wray 977 , has a mass within $39-53 M_{\odot}$ and a radius of $62 R_{\odot}$, close to the Roche lobe radius near periastron (Kaper et al. 2006). The X-ray emission of GX 301-2 exhibits periodic flares about 1.4 days before the periastron passage (e.g. Sato et al. 1986), and various models, such as equatorial disk and tidal stream, have been proposed to explain the pre-periastron flare (Pravdo \& Ghosh 2001; Leahy \& Kostka 2008). Mönkkönen et al. (2020) discovered that the spin frequencies of GX 301-2 show an orbital spinup to spin-down reversal near periastron. If this orbital spin reversal is caused by passage of a tidal stream over the neutron star, as modelled by Leahy \& Kostka (2008), the neutron star should rotate in the opposite direction of the orbital revolution (Mönkkönen et al. 2020).

Because a case of a retrograde neutron star will have

(C) 0000 RAS 

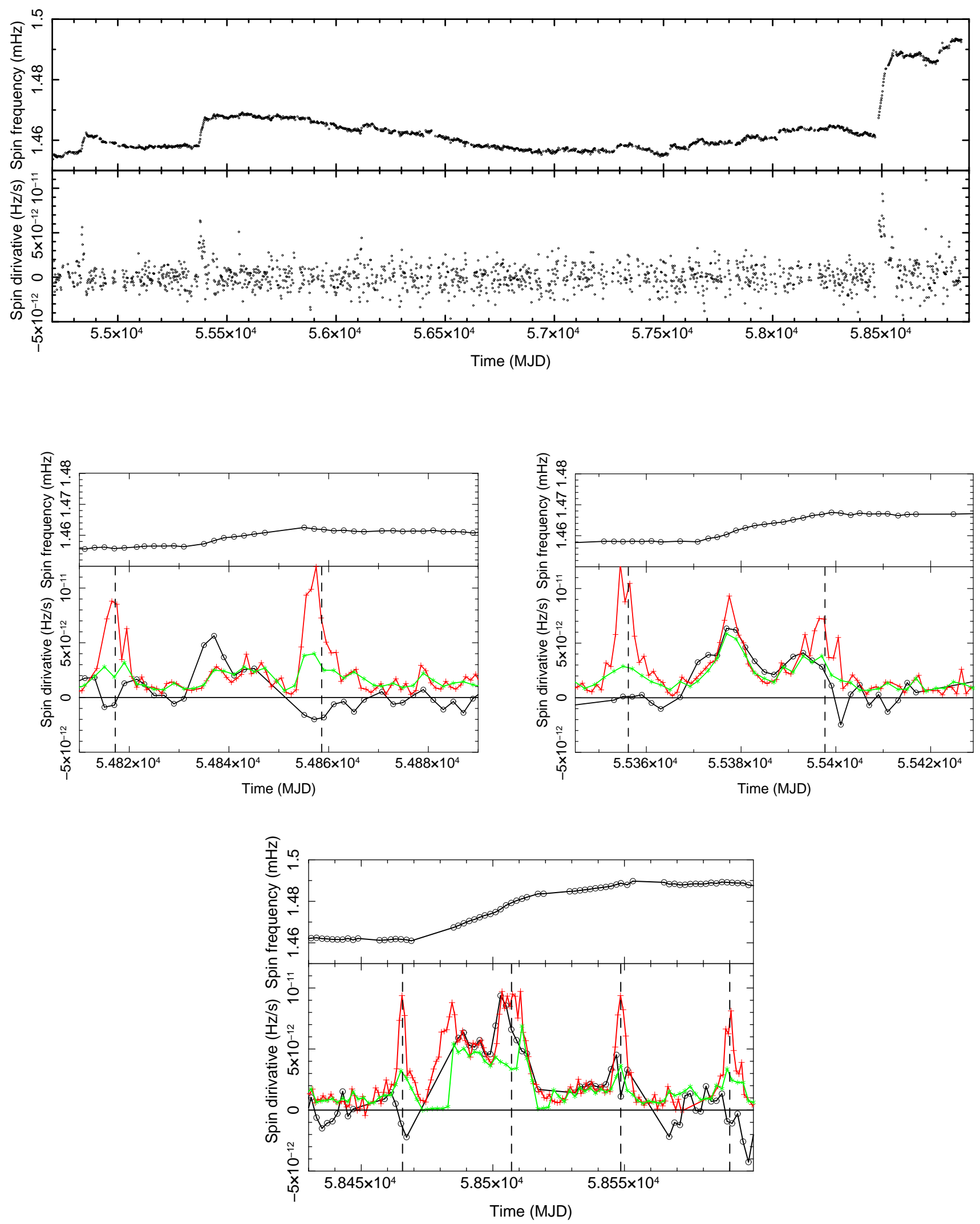

Figure 1. Top: the spin history of GX 301-2 monitored by Fermi/GBM since 2008-8-13. Three rapid spin-up events are prominent around MJD 54850, 55400, and 58500. The corresponding spin derivatives are over-plotted. Middle and bottom: zoomed plot of the three spin-up events, together with the Swift/BAT 15-50 keV fluxes (red pluses) and the pulsed fluxes within 12-50 keV estimated by Fermi/GBM (green stars). Vertical dashed lines indicate the time of periastron. 
profound implications for the understanding of X-ray pulsar, in this paper, we study the rare spin-up events of GX 3012 , which also provide insights on the spin direction of GX 301-2. The pulse frequency history of GX 301-2 monitored by the Burst and Transient Source Experiment (BATSE) on the Compton gamma ray observatory revealed two rapid spin-up events, each lasting for about 30 days, besides erratic variations (Koh et al. 1997). The Gamma-ray Burst Monitor (GBM, Meegan et al. 2009) on the Fermi spacecraft detected other three rapid spin-up events, with the most powerful one occurred in Jan., 2019. We will study in detail the three rapid spin-up events detected by Fermi/GBM, together with the data from Swift/BAT and MAXI. The last spin-up event has been reported by Nabizadeh et al. (2019) and Abarr et al. (2020). We will also show that the flare of intrinsic X-ray emission (15-50 keV) of GX 301-2 happened 0.4 days before periastron, different from the flare of low energy emission (2-10 keV), which happened about 1.4 days before periastron.

\section{SPIN-UP EVENTS OF GX 301-2}

The spin histories of X-ray pulsars are continuously monitored currently with Fermi/GBM 1 (Finger et al. 2009; Malacaria et al. 2020). The spin frequencies $(\nu)$ of GX 3012 monitored by Fermi/GBM are presented in Figure 1, together with the spin derivative, $\dot{\nu}$, calculated with a running three points derivative. As can be seen, the spin frequencies vary on a timescale of days, with three rapid spin-up events around MJD 54850, 55400, and 58500. The spin derivatives vary around 0 , with an average amplitude around $1 \times 10^{-12}$ $\mathrm{Hzs}^{-1}$. The three rapid spin-up events are zoomed in the middle and bottom panel of Figure 1. The corresponding 15$50 \mathrm{keV}$ fluxes measured by Swift/BAT and 12-50 keV pulsed fluxes estimated with Fermi/GBM are also presented in the zoomed plots. The Swift/BAT data are taken from Swift hard X-ray transient progran 2. As Fermi/GBM searches the pulsar periodic signal using Fourier method due to the strong background, only the pulsed flux is obtained.

The first spin-up event around MJD 54850 lasts for about 20 days, the second event around 55400 lasts for about 30 days, while the last one around 58500 lasts for about 80 days. As can be seen from the zoomed plot, except for the first 10 days of the first event and the periastron period of the last event, the spin derivatives during the spin-up periods are well correlated with the Swift/BAT fluxes. To further illustrate this point, in figure 2, we plot the relation of spin derivative vs Swift/BAT flux for the three spin-up periods, and for the no spin-up period between the second and the last spin-up events (MJD 55400-58400). For this purpose, the Swift/BAT fluxes are obtained as the mean value of the orbital light curves during the times within which the spin derivatives are calculated. We see that the spin derivatives within the no spin-up period show no correlation with the fluxes, while those during the three spin-up episodes stand out differently and show a positive correlation with the fluxes. On the other hand, different from a

\footnotetext{
1 https://gammaray.msfc.nasa.gov/gbm/science/pulsars/

2 https://swift.gsfc.nasa.gov/results/transients/
}

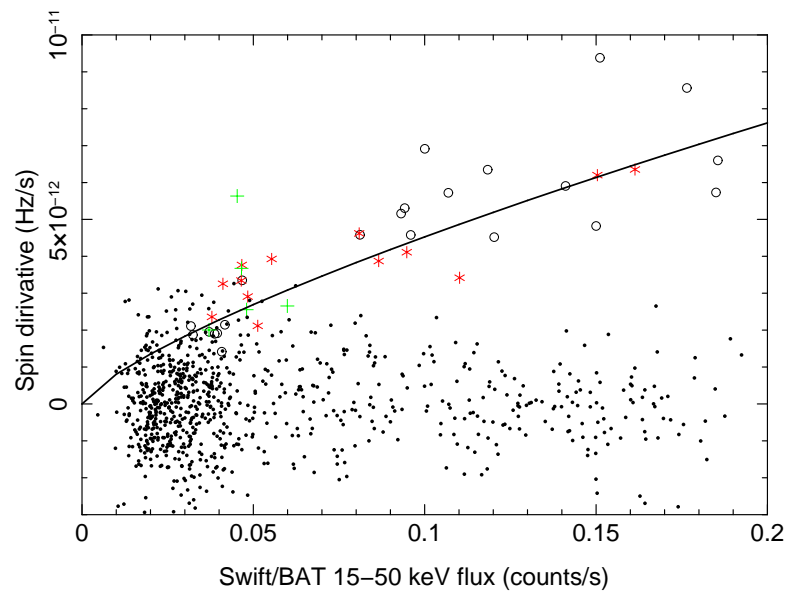

Figure 2. The relation of spin derivatives $(\dot{\nu})$ vs Swift/BAT 15$50 \mathrm{keV}$ fluxes for the first (green pluses), the second (red stars), and the last (open circles) spin-up events. The relation during the no spin-up period (MJD 55400-58400) are over-plotted as black dots. The spin derivatives are correlated with the fluxes for the spin-up events, with a fitted relation of $\dot{\nu} \propto F^{0.75 \pm 0.05}$ (solid line).

well correlation of pure disk accretion (e.g., A0535+262 and GRO J1744-28, Bildsten et al. 1997), the data of the spinup events of GX 301-2 are more scattered, which could be due to contamination of wind accretion. We fit a power-law to the spin derivatives during spin-up episodes and obtain a relation of $\dot{\nu} \propto F^{0.75 \pm 0.05}$ for $90 \%$ confidence level assuming that the measured derivatives have 10 percent errors.

At the beginning of the last spin-up event, the pulsed fluxes are much lower than normal values for about 10 days, and the spin frequencies are not measurable by Fermi/GBM. Indeed, as shown by Abarr et al. (2020), during this period, the pulse fraction within 15-35 keV measured with $\mathrm{X}$-Calibur is only $\sim 30 \%$. They noted that the shape of the $\mathrm{X}$-Calibur $15-35 \mathrm{keV}$ pulse profile is dominated by one peak, quiet different from the normal two-peak profiles observed by $N u S T A R$ during other periods, and these features can be caused by changes of the accretion and emission geometries. The column density during this low-pulse-fraction period measured by Abarr et al. (2020) are around $8 \times 10^{23} \mathrm{~cm}^{-2}$, higher than the average value measured around these phases $\left(\sim 10^{23} \mathrm{~cm}^{-2}\right.$, e.g. Islam \& Paul 2014). It indicates that significant material is present around the neutron star during this low-pulse-fraction period.

As can be seen from the zoomed plot, all three events started about 10 days after the periastron, a feature already noted by Abarr et al. (2020). Similar starting time was also true for the two spin-up events reported by Koh et al. (1997). The meaning of this start time will be discussed in $\S .4$.

\section{3 "PRE"-PERIASTRON FLARE OF GX 301-2}

Early observations of GX 301-2 revealed that the Xray fluxes of GX 301-2 show an orbital flare about 1.4 days before the periastron passage (e.g. Sato et al. 1986), a very 


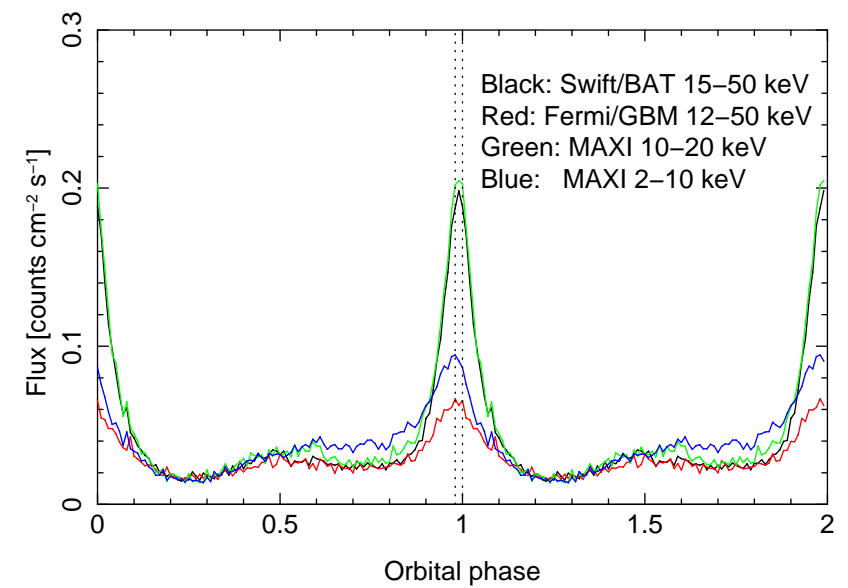

Figure 3. Orbital profiles of the fluxes of Swift/BAT 15-50 keV (black), MAXI 10-20 keV (green), MAXI 2-10 keV (blue), and the pulsed fluxes of Fermi/GBM within $12-50 \mathrm{keV}$ (red). Two dotted lines around phases of 0.98 and 1 are plotted to guide eyes.

particular feature among X-ray pulsars. Many continuous monitoring of GX 301-2 in different energy bands are available in recent years, such as Swift/BAT and MAXI, and they allow more detailed studies of the behavior of the preperiastron flare of GX 301-2. In Figure 3, we plot the orbital profile of the fluxes of Swift/BAT 15-50 keV, MAXI 2-10 keV and 10-20 keV, and of the pulsed fluxes within 12-50 keV estimated by Fermi/GBM. The MAXI data are taken from its Gas Slit Camerd? We use the orbital parameter of period $P=41.472$ days with $\dot{P}=-3.7 \times 10^{-6}$, and a periastron time $T_{\text {per }}=53531.65$ (MJD) given by Doroshenko et al. (2010). The no spin-up period between MJD 55400 and 58400 is adopted. All the profiles are normalized to the Swift/BAT profile at phase 0.5, and 100 orbital phase bins are used.

It can be seen that the Swift/BAT 15-50 keV and MAXI 10-20 keV profiles are quite similar, both of which show a prominent peak around phase 0.99 , only 0.4 days before the periastron passage. The peak fluxes are about 10 times the lowest values around phases between 0.2 and 0.3 . The MAXI $2-10 \mathrm{keV}$ profile is peaked around phase 0.98 , but it is skewed to earlier phases, with a centroid around phase 0.97 . The peak $2-10 \mathrm{keV}$ fluxes are about 4.5 times the lowest values. In contrast, the peak pulsed fluxes within $12-50 \mathrm{keV}$ are only 3 times the lowest values, indicating that the pulsed fractions around the flux peak are only $30 \%$ of those around the low flux periods. These results show that the intrinsic high-energy X-ray emission (15-50 keV) of GX 301-2 peaked around orbital phase 0.99 , very close to the periastron passage, and the "pre"-periastron flare is only significant for lower energies (2-10 keV), which are more easily absorbed by matter around GX 301-2.

The drop of pulse fraction near periastron has already been shown by Nabizadeh et al. (2019), and they noted that it may be caused by scattering in the dense wind environment. To further illustrate the nature of the drop of pulsed

3 http://maxi.riken.jp/

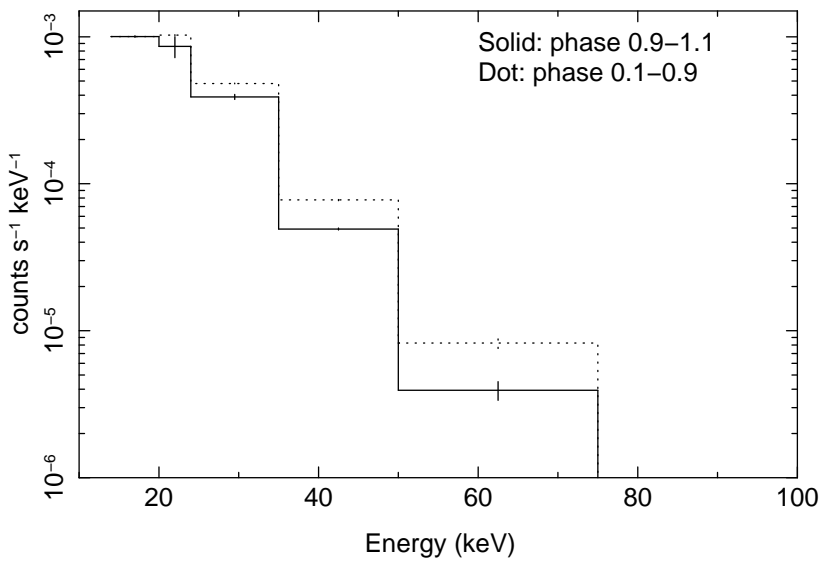

Figure 4. Swift/BAT spectra of GX 301-2 extracted from phases within 0.9-1.1 (near periastron) and with 0.1-0.9. The steeper spectral shape of the spectrum within phase 0.9-1.1 indicates Compton scattering of the intrinsic high energy X-ray emission near periastron.

fraction near periastron, we extract the averaged Swift/BAT spectra for orbital phases within 0.1-0.9 and 0.9-1.1, respectively, using the snapshot light curves of the eight bands of BAT. The spectra are presented in Figure 4, and the spectrum within phase $0.1-0.9$ is normalized to that of phase 0.9-1.1 at 14-20 keV. It can be seen that the spectrum near periastron is steeper than that away from periastron. That is, if the intrinsic X-ray emission is similar for all phases, the higher energy photons near periastron are more scattered to lower energies. This is the typical behavior of Compton scattering process. Indeed, Compton shoulder of the Fe $\mathrm{K} \alpha$ line has been reported for GX 301-2 with Chandra observations near periastron (Watanabe et al. 2003; Liu et al. 2018). Considering the absorption column density of GX 301-2 near periastron is generally larger than $10^{24} \mathrm{~cm}^{-2}$, the Compton scattering optical depth is larger than 1, a large part of the intrinsic high energy emission will be scattered, and the observed high energy X-ray photons will also contain a large amount of scattered photons. The surface of the optical star could also be an origin of the scattering medium. Therefore, Compton scattering will reduce the pulse fraction near periastron, just as observed. Compton reflection might also affect the measurement of the spin derivatives near periastron, which will be discussed in next section.

\section{CONCLUSION AND DISCUSSION}

We studied the three rapid spin-up events of GX 3012 detected by Fermi/GBM in last ten years together with Swift/BAT and MAXI data. The spin derivatives of GX 301-2 during the no spin-up period show no correlation with the Swift/BAT 15-50 keV fluxes, consistent with a wind accretion mode; while the spin derivatives during the rapid spin-up periods are correlated with the Swift/BAT fluxes, following a relation of $\dot{\nu} \propto F^{0.75 \pm 0.05}$. Such a correlation shows that the accreted matter has the same flow direction as the rotation of the neutron star, consistent with accretion of a prograde disk to the neutron star (Pringle \& Rees 1972; 
Rappaport \& Joss 1977). These results confirm the previous idea that the rare spin-up events of GX 301-2 are due to a transient disk (Koh et al. 1997).

All the rapid spin-up events of GX 301-2 started about 10 days after the periastron passage. We note that such a timescale is corresponding to the time needed for the tidally stripped gas to reach the neutron star. As shown in hydrodynamic simulations by Lavton et al. (1998), the tidally stripped gas reaches the neutron star around phase 0.25 , just about 10 days after the periastron passage. The optical star Wray 977 has a radius of $62 R_{\odot}$, while the Roche-Lobe radius near periastron is about $66 R_{\odot}$ estimated with the formula given by Sepinsky et al. (2007). As a result, Wray 977 is underfill its Roche-Lobe radius, consistent with the fact that GX 301-2 is dominated by wind accretion. Nevertheless, the radius of Wray 977 is close enough to the Roche-Lobe radius near periastron, making it possible to transport some tidally stripped gas to the neutron star occasionally, to produce the observed spin-up events. Such a transport process might be caused by the instability of Wray 977 or other unknown effects.

Optical spectral observations of Wray 977 favor a slow rotation of Wray 977, with a period comparable to the orbital period of GX 301-2 (Parkes et al. 1980; Kaper et al. 2006). Therefore, Wray 977 is rotating much slower $(\sim 1 / 3)$ compared to the angular velocity of the neutron star near periastron. The simulation by Layton et al. (1998) predicted a spin-down event following the spin-up event, which is not observed for the spin-up events (but Mönkkönen et al. (2020) discovered an orbital spin-up to spin-down reversal near periastron). The predicted spin-down is due to their assumption of synchronous rotation of the optical star near periastron, which makes the stripped gas rotating faster than the angular velocity of the neutron star after periastron and leads to a reversed accretion direction after the stripped stream passes over the neutron star. The slow rotation of Wray 977 makes the passage of stripped gas stream over the neutron star unlikely, consistent with the fact that only spin-up events are observed.

For a scenario of accretion of tidally stripped gas to the neutron star, the stripped matter generally has angular momentum in the direction of the orbital motion, as in a Roche-Lobe-like overflow. The stripped matter might pass over the neutron star only when it obtains a high radial velocity and when the optical star rotates faster than the angular velocity of the neutron star near periastron. The slow rotation nature of Wray 977 makes a retrograde disk unlikely. Therefore, the observed spin-up events and the slow rotation of GX 301-2 favor a prograde neutron star of GX 301-2. This is in contrast to the idea of a retrograde neutron star proposed by Mönkkönen et al. (2020) to explain the orbital spin-up to spin-down reversal near periastron. Their idea is based on the stream model invoked to explain the pre-periastron flare of GX 301-2 (Leahy \& Kostka 2008). We found that the flare of the intrinsic X-ray emission (high energy) of GX 301-2 happened around phase 0.99, only 0.4 days before the periastron, while the flare of low energies (2-10 keV) happened about 1.4 days before the periastron. The preceding low-energy flare can be explained as due to stronger absorption of the intrinsic X-ray emission closer to the periastron. This finding weakened the need of the stream model for GX 301-2. In addition, the stream model requires that the optical star rotates twice faster as the orbital motion, in conflict with the slow rotation of Wray 977.

The pulse fraction of GX 301-2 near periastron is reduced heavily (30\%) compared with other orbital phases. Such a drop of pulse fraction can be explained by Compton scattering from the dense material around Wray 977 and/or from the surface of Wray 977. Compton process may also provide a clue to the orbital spin reversal near periastron discovered by Mönkkönen et al. (2020). The periastron distance between the neutron star and the surface of Wray 977 is only about $30 R_{\odot}(70 \mathrm{lt}-\mathrm{s})$, while around phase 0.9 the distance is about $80 R_{\odot}(185 \mathrm{lt}-\mathrm{s})$. These distances are smaller than the light travel distances within the pulse period of GX 301-2, 680 s. Therefore, Compton reflected photons from the optical companion might affect the observed pulse profile heavily and affect the measurement of spin derivatives near periastron. Detailed modelling of reprocession and transport of hard X-ray emission is needed to test whether Compton reflection is responsible for the observed orbital spin reversal of GX 301-2.

\section{ACKNOWLEDGEMENTS}

I thank the referee for helpful comments, Jenke Peter A. for help on Fermi/GBM data, Lien Amy Yarleen for help on Swift/BAT data, Zheng Xueying, Liao Zhenxuan, Lu Youjun, Li Xiangdong, and Epili Prahlad for helpful discussions. This research is supported by National Natural Science Foundation of China (11773035 and U1938113) This research used data obtained with the Fermi/GBM, Swift/BAT, and MAXI.

\section{DATA AVAILABILITY}

The data underlying this article are publicaly available from the web as listed in the footnote.

\section{REFERENCES}

Abarr, Q. et al. 2020, ApJ, 891, 70

Bildsten, L. et al. 1997, ApJS, 113367

Brandt, N. \& Podsiadlowski, P. 1995, MNRAS, 274, 461

Chakrabarty, D. et al. 1997, ApJ, 474, 414

Doroshenko, V.; Santangelo, A.; Suleimanov, V.; Kreykenbohm, I.; Staubert, R.; Ferrigno, C.; Klochkov, D. 2010, A\&A, 515, 10

Finger, M. H. et al. 2009, arXiv0912.3847

Ghosh, P. \& Lamb, F. K. 1979, ApJ, 234, 296

Hills, J. G. 1983, ApJ, 267, 322

Islam, N. \& Paul, B. 2014, MNRAS, 441, 2539

Kaper, L.; van der Meer, A.; Najarro, F. 2006, A\&A, 457, 595

13. Koh, D. T.; Bildsten, L.; Chakrabarty, D. 1997, ApJ, 479, 933

Leahy, D. A. \& Kostka, M. 2008, MNRAS, 384, 747

Liu, J., Soria, R., Qiao, E., Liu, J., 2018, MNRAS, 480, $4746 \mathrm{~L}$

Mönkkönen, J., Doroshenko, V., Tsygankov, S. S., Nabizadeh, A., Abolmasov, P., Poutanen, J. 2020, MNRAS, 494.2178 
Makishima, K. et al. 1988, Nature, 333, 746

Malacaria, C., Jenke, P., Roberts, O. J., Wilson-Hodge, C. A., Cleveland, W. H., Mailyan, B. 2020, arXiv:2004.00051 Meegan, C. et al. 2009, ApJ, 702, 791

Mukherjee, U.; Paul, B. 2004, A\&A, 427, 567

Nabizadeh, A.; Mönkkönen, J.; Tsygankov, S. S.; Doroshenko, V.; Molkov, S. V.; Poutanen, J. 2019, A\&A, 629, 101

Parkes, G. E., Mason, K. O., Murdin, P. G., Culhane, J. L. 1980, MNRAS, 191, 547

Pravdo, S. H.; Ghosh, P. 2001, ApJ, 554, 383

Pringle, J. E.; Rees, M. J. 1972, A\&A, 21, 1

Rappaport, S. \& Joss, P. C. 1977, Nature, 266, 683

Sato, N.; Nagase, F.; Kawai, N.; Kelley, R. L.; Rappaport, S.; White, N. E. 1986, ApJ, 304, 241

Sepinsky, J. F., Willems, B., Kalogera, V. 2007, ApJ, 660, 1624

Layton, J. T.; Blondin, J. M.; Owen, M. P.; Stevens, I. R. 1998, NewA, 3, 111

Wang, Y. M. 1981, A\&A, 102, 36

Watanabe S., et al., 2003, ApJ, 597, L37

White, N. E.; Mason, K. O.; Huckle, H. E.; Charles, P. A.; Sanford, P. W. 1976, ApJ, 209, L119 